\title{
DEVELOPMENT OF DIFFRACTION RADIATION DIAGNOSTICS FOR NONINVASIVE BEAM SIZE, DIVERGENCE, AND EMITTANCE MEASUREMENTS
}

\author{
W. D. Kimura, ${ }^{\#}$ STI Optronics, R. B. Fiorito, Catholic U. America, and D. W. Rule, NSWCCD
}

\begin{abstract}
The analysis and design for a nondestructive electron beam diagnostic that is based upon observing diffraction radiation (DR) is presented. DR is similar to transition radiation and is produced when the beam passes through an aperture or near an edge. Analysis of the DR permits a number of beam parameters to be determined, such as the beam's transverse size, longitudinal (temporal) distribution, divergence, emittance, and energy. Our analysis includes a procedure for separating out this information from the DR angular distribution pattern. This analysis then forms the basis for the design of a relatively simple and compact aperture or slit system that can be used to nondestructively measure these parameters in real time.
\end{abstract}

\section{INTRODUCTION}

Electron linear accelerators (linacs) are used in a wide variety of applications including scientific, medical, and industrial. New applications are emerging such as highpower self-amplified spontaneous emission (SASE) [1] free electron laser (FEL) devices and high-duty-cycle FELs for industrial processing [2]. These devices require electron beams ( $e$-beams) with very high quality (i.e., low emittance), and accurate position and size control. The small beam size and high average power of these accelerators preclude the use of conventional interceptive diagnostics and make it very difficult to monitor the beam quality on line.

The ultimate goal of our program is to develop a suite of noninterceptive diffraction radiation (DR) diagnostics capable of measuring beam divergence, energy, position, transverse beam size, emittance and bunch length. These DR diagnostics will minimally perturb the beam, will respond rapidly to changes in the beam so that the beam can be tuned while its characteristics are being measured in real time, and will easily interface with automated computer-controlled data acquisition and feedback systems. Our approach features a compact design that is capable of simultaneously measuring multiple beam parameters at one position along the beamline.

\section{REVIEW OF DR THEORY}

DR is produced when an electron passes through an aperture or near an edge, e.g., a slit. It is closely related to transition radiation (TR) with similar behavior. However, the development of DR theory is still quite sparse compared with TR and even less experimental work on DR has been done [3].

Appreciable DR is generated when the condition $\gamma \hat{\lambda} \geq$ $a$ is satisfied, where $\gamma=$ Lorentz factor, $\hat{\lambda}=$ DR wavelength $/ 2 \pi$, and $a=$ aperture radius. As illustrated in Fig. 1, a convenient geometry is to have the aperture inclined at $45^{\circ}$ to the $e$-beam so that the backward DR light is directed orthogonal to the $e$-beam towards detectors.

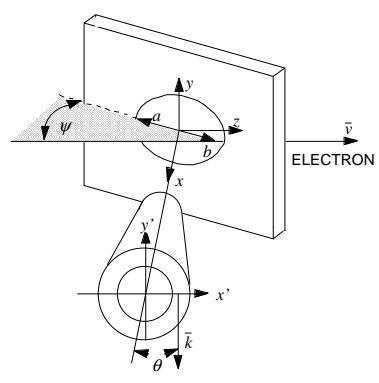

Figure 1: DR emission geometry for $45^{\circ}$ aperture.

For the case of a circular aperture the DR photon production is given by [4]

$$
\frac{\mathrm{d}^{2} I_{D R}}{\mathrm{~d} \omega \mathrm{d} \Omega}=\frac{\mathrm{d}^{2} I_{T R}}{\mathrm{~d} \omega \mathrm{d} \Omega} B(N, \bar{k}, \ell, \sigma) G(q, a)
$$

where $\omega=$ frequency, $\Omega=$ solid angle, $N=$ number of electrons, $k=2 \pi / \lambda, \sigma=$ beam size, $q=k \sin \theta, \theta=$ observation angle, and $a=$ aperture radius. The TR term goes as

$$
\frac{\mathrm{d}^{2} I_{T R}}{\mathrm{~d} \omega \mathrm{d} \Omega} \approx \frac{e^{2}}{\pi^{2} c} \frac{\theta^{2}}{\left(\gamma^{-2}+\theta^{2}\right)^{2}}, \gamma>>1,
$$

\footnotetext{
*Work supported by U.S. Department of Energy, Grant No. DE-FG03-98ER82673.

\#Email: wkimura@stioptronics.com
} 


$$
\begin{aligned}
& B(N, \bar{k}, \ell, \sigma)=N+N(N-1) F(\bar{k}, \ell, \sigma), \\
& F(\bar{k}, \ell, \sigma)=\left|\int S(\bar{r}) e^{i k \bullet \bar{r}} \mathrm{~d} \bar{r}\right|^{2},
\end{aligned}
$$

where $S(\bar{r})=$ beam distribution function. Note, when $N$ $>N_{\mathrm{c}} \equiv(1-F) / F$, then coherent radiation dominates. Lastly, the geometrical factor for a circular aperture is

$$
G(q, a)=J_{0}^{2}(k a \sin \theta)+(b / a)^{2} J_{1}^{2}(k a \sin \theta),
$$

where $b$ is the offset from the center of the aperture and $J_{0,1}$ are Bessel functions. Thus, the aperture modifies the normal TR geometrical factor through the addition of a $J_{1}^{2}$ term.

An example of the amount of DR photons generated from a 2-mm dia. aperture is shown in Fig. 2 for conditions similar to the $6-\mathrm{GeV}$ beam at the Thomas Jefferson National Accelerator Facility (TJNAF).

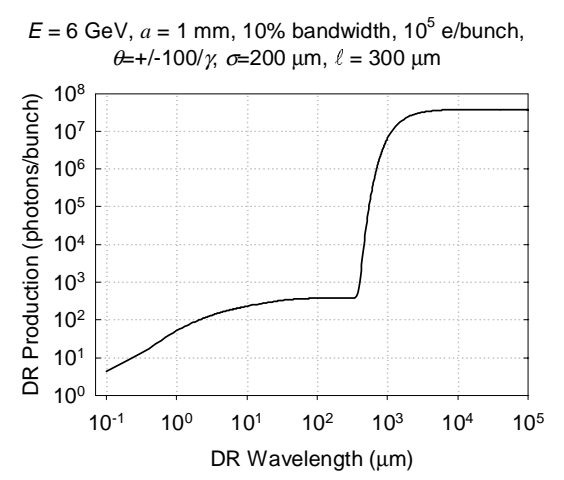

Figure 2: Example of DR production from circular aperture.

The effect of coherence can be clearly seen where the photon number increases dramatically at wavelengths $\geq 300 \mu \mathrm{m}$, which corresponds to the bunch length $\ell$.

\section{DIAGNOSING WITH DR}

The DR observables are: 1) near-field and far-field intensity, 2) polarization, 3) angular distribution in farfield, and 4) wavelength spectrum. Using these observables, the beam offset, size, divergence, and bunch length can be measured.

For example, the beam divergence and size can be separately determined for the circular aperture by observing the far-field angular distribution pattern using appropriate choices of aperture size and observation wavelength for a given beam energy [5]. We have recently shown that this can also be accomplished using a slit DR radiator by separately observing the perpendicular and parallel components of the far-field angular distribution pattern. As an example, for a slit the perpendicular polarization pattern is more sensitive to changes in beam size than the parallel polarization. This is illustrated in Fig. 3 for a beam with size $\sigma=200 \mu \mathrm{m}$. As can be seen there are more DR photons at the center of the pattern for the perpendicular component than for the parallel component.

With the beam size and divergence it is possible to determine the beam emittance. This ability to separate the divergence and size information from the angular distribution pattern by using polarization is a new way of using DR as a diagnostic tool.

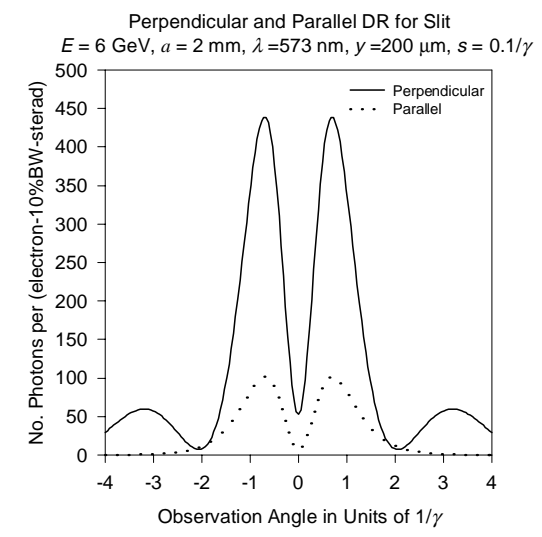

Figure 3: DR production from a 2-mm wide slit.

Our calculations have shown that a single slit DR diagnostic should be capable of resolving divergence angles $\sim 0.1 / \gamma$ and beam sizes $\sim 50 \mu \mathrm{m}$. We are currently exploring ways to improve and extend the sensitivity of the measurements. For example, using double apertures can help further increase the sensitivity to beam divergence. In a dual-aperture interferometer, as depicted in Fig. 4, the backward and forward DR emission interfere with each other. For this to occur the distance between apertures must be greater than or equal to the forward coherence length $L_{\mathrm{c}}=\gamma^{2} \lambda$.

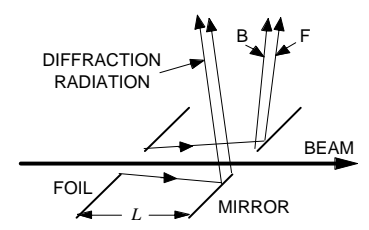

Figure 4: Dual aperture DR interferometer.

This interference between the forward and backward DR causes an additional modulation on the angular distribution as shown in Fig. 5 for the case of $L=6 L_{\mathrm{c}}$. Note, how divergence angles of $\sim 0.05 / \gamma$ can be resolved.

Another new development is using the near-field intensity distribution around the perimeter of the aperture to determine information about the beam position and profile. For example, as the beam moves away from the center towards one side of the aperture, increased DR emission will occur from that side and less from the side $180^{\circ}$ away. An example of this is shown in 
Fig. 6, which plots the DR intensity distribution around the perimeter for a 2-mm dia. aperture at offsets of $X_{0}=$ 0 and $200 \mu \mathrm{m}$ from the center.

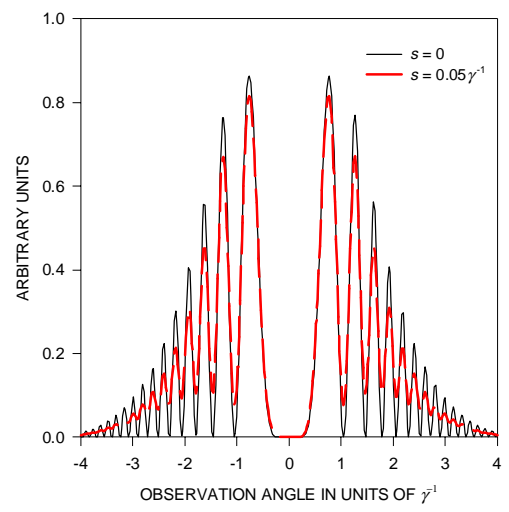

Figure 5: DR angular distribution pattern for dual apertures and beam divergence $s=0$ and $s=0.05 / \gamma$.

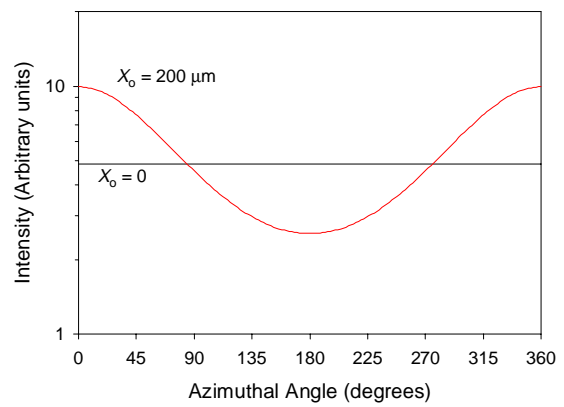

Figure 6: Near-field DR intensity distribution for a Gaussian beam $(\sigma=200 \mu \mathrm{m})$ offset from the center by $X_{0}$.

Through this analysis we have found that for the same parameters used in Fig. 2 (i.e., TJNAF values), a 10- $\mu \mathrm{m}$ offset of the beam centroid will cause a normalized peak-to-peak difference (i.e., $\Delta I / I$ ) variation of the sinusoid curve shown in Fig. 6 of 7\%. Since variations of a few percent should be discernible, this implies $<10$ $\mu \mathrm{m}$ offsets can be detected. Hence, this diagnostic has the capability of being a high resolution beam position monitor (BPM).

Similarly, if the beam is ellipsoidal in shape, the sinusoid curve in Fig. 6 will have a pair of peaks and valleys whose height and depth depends on the aspect ratio $\left(\sigma_{x} / \sigma_{y}\right)$ of the ellipticity. Again, for the TJNAF conditions we find that an aspect ratio of $\sigma_{x} / \sigma_{y}=0.9$ corresponds to an intensity contrast of $\Delta I / I \approx 5 \%$. Thus, $\sim 10 \%$ changes in aspect ratio should be discernible.

More details of the beam profile may be obtainable by applying the theory of moments [6] to the distribution of the near-field pattern.

As mentioned earlier, the bunch length can be directly measured by examining at what wavelength the coherent production of DR occurs as illustrated in Fig. 2.

\section{DR DIAGNOSTIC SYSTEM}

A DR diagnostic system is conceptually very simple in design and can be compact in size. An example of a general purpose DR diagnostic system is illustrated in Fig. 7. It has a moveable DR target, which might consist of a combination of apertures, slits, and/or single knife edges. This target is held at $45^{\circ}$ with respect to the $e$ beam. A beamsplitter located below the beamline directs the DR light to a pair of high-sensitivity, lownoise CCD cameras. One camera images the near-field; the other images the far-field. Before the cameras are pairs of wheels containing polarizers and bandpass filters. The output from the cameras are sent to a PC for data reduction and analysis. Lead surrounds the cameras to minimize radiation-induced noise.

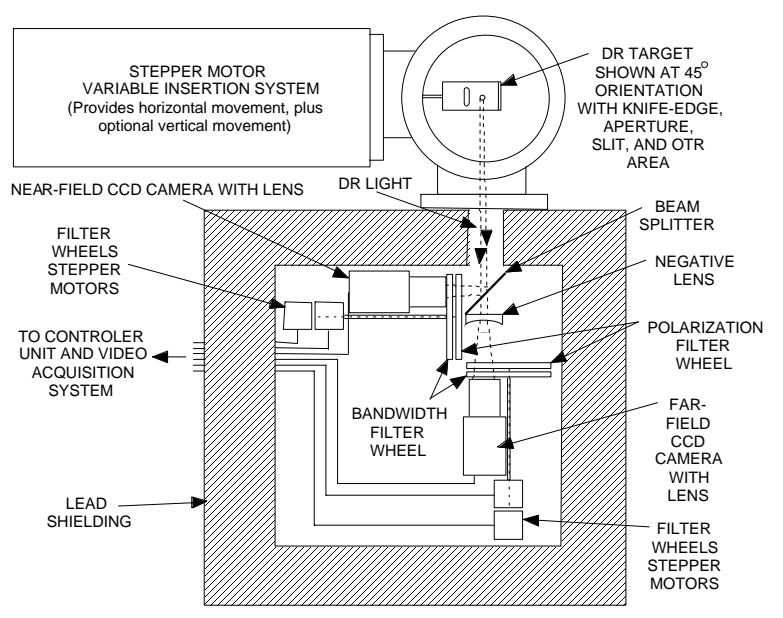

Figure 7: DR diagnostic system layout.

\section{CONCLUSIONS}

Our analysis has shown the viability of $D R$ as a noninterceptive beam diagnostic tool and quantified its capabilities. We have also shown the radiative power loss caused by the aperture is minuscule $(\approx 1 \mathrm{~mW}$ for a 6 $\mathrm{GeV}, 200 \mu \mathrm{A}$ beam), the voltage stresses induced on the aperture edges by the self-fields of the electrons are small, and the wakefield effects on the beam for the TJNAF conditions are negligible.

\section{REFERENCES}

[1] Tenth Advanced ICFA Beam Dynamics Panel Workshop on 4th Generation Light Sources, Grenoble, France, Jan. 22-25, 1996.

[2] M.J. Kelley, H.F. Dylla, G.R. Neil, L.J. Brillson, D.P. Henkel, and H. Helvajian, SPIE 2703, 15 (1996).

[3] See for example, Y. Shibata, et al., Phys. Rev. E 52, 6787 (1995).

[4] D. W. Rule, R. B. Fiorito, W. D. Kimura, "Noninterceptive Beam Diagnostics Based on Diffraction Radiation," 7th Beam Instrumentation Workshop, Argonne, IL, May 6-9, 1996.

[5] R. B. Fiorito, D. W. Rule, and W. D. Kimura, "Noninvasive Beam Position, Size, Divergence, and Energy Diagnostics Using Diffraction Radiation," in Proceedings of 8th Workshop on Advanced Accelerator Concepts, Baltimore, MD, Jul. 5-11, 1998.

[6] M. R. Teague, J. Opt. Soc. Am. 70, 920 (1980). 